\title{
Atributos contextuales influyentes en el proceso de educción de requisitos: una exhaustiva revisión de literatura
}

\author{
Influential contextual attributes in the requirements elicitation process: \\ a comprehensive literature review
}

Dante Carrizo Moreno ${ }^{1}$

Recibido 24 de abril de 2014, aceptado 22 de julio de 2014

Received: April 24, $2014 \quad$ Accepted: July 22, 2014

\begin{abstract}
RESUMEN
La educción de requisitos captura información relevante para la conformación de los requisitos del software mediante el uso de una gran cantidad de técnicas. Sin embargo, la efectividad del proceso depende del contexto en que este ocurre. Este artículo pretende dar a conocer los atributos del contexto que influyen en el proceso de educción. Para ello, se realiza una revisión sistemática y no sistemática de la literatura científica para determinar cuáles son estos atributos. El resultado de esta revisión arrojó 118 atributos que se reagruparon en 27 atributos genéricos correspondientes a 5 factores: del eductor, del informante, del dominio del problema, del dominio de la solución y del proceso mismo. Este estudio contribuye a orientar futura investigación empírica sobre la educción de requisitos señalando los posibles factores experimentales a considerar.
\end{abstract}

Palabras clave: Ingeniería de requisitos, educción de requisitos, técnicas de educción, atributos contextuales, revisión sistemática.

\section{ABSTRACT}

The requirements elicitation captures relevant information to the shaping of software requirements by using several techniques. However, the effectiveness of the process depends on the context in which it occurs. This article aims to reveal the attributes of the context influencing the elicitation process. For this purpose, a systematic and non-systematic review of the scientific literature was conducted in order to determine which are these attributes. The result of this review yielded 118 attributes that were grouped into 27 generic attributes corresponding to 5 factors: the eductor, the informant, the problem domain, the solution domain and the process itself. This study helps to guide future empirical researches on requirements elicitation pointing out possible experimental factors to consider.

Keywords: Requirements engineering, requirements elicitation, elicitation techniques, contextual attributes, systematic review.

\section{INTRODUCCIÓN}

El proceso de requisitos (IR) comprende actividades como: educción, análisis, especificación, validación y gestión de requisitos [1]. La educción de requisitos, en particular, trata la captura y descubrimiento de las necesidades de los stakeholders. Su objetivo es: identificar información acerca del dominio del problema, lo que ayuda a que los ingenieros de requisitos adquieran conocimiento del mundo del usuario; y determinar las propiedades deseadas del sistema software.

1 Departamento Ingeniería Informática y Ciencias de la Computación. Universidad de Atacama. Avenida Copayapu 485. Copiapó, Chile. E-mail: dante.carrizo@uda.cl 
Para capturar la información relevante los analistas, muy a menudo, utilizan únicamente entrevistas [2]. Sin embargo, hay otras técnicas de educción que también pueden ser utilizadas para esta actividad. Algunas revisiones dan cuenta de decenas de técnicas [3]. Muchas de estas técnicas han sido adoptadas de otras disciplinas como la psicología cognitiva, la antropología, la sociología o la lingüística [4], y han sido exitosamente utilizadas en ingeniería del conocimiento y, posteriormente, en ingeniería de software [5].

Es probable que más de una técnica de educción sean necesarias para obtener el rango completo de requisitos para la mayoría de los sistemas software complejos. Debido a la diferencia de naturaleza de las técnicas de educción [6], es posible esperar que sus desempeños sean mejores en unas situaciones que en otras. Es decir, determinados atributos del contexto del proyecto influyen en el comportamiento de las técnicas de educción y, por lo tanto, en la efectividad del proceso [7]. Los atributos contextuales tienen que ver con aspectos del entorno del proceso de educción. No se refieren a características intrínsecas de las técnicas sino más bien de los agentes del contexto. Es la variación de estas características de los aspectos contextuales las que prescriben las facetas propias que deberían poseer las técnicas para que su uso sea adecuado.

De esta manera, la determinación de los atributos contextuales que influyen en la efectividad de las técnicas de educción es una necesidad prioritaria para llegar a diferenciar la adecuación de las técnicas y poder seleccionar acertadamente la que puede ser más efectiva en una sesión de educción. La influencia del contexto en la efectividad de las técnicas de educción ha sido enunciada en literatura de requisitos [7-11]. Estas opiniones se asientan en la experiencia y conocimiento adquirido por cada autor. Sin embargo, sería deseable la validación empírica de estas aseveraciones. Esta evaluación puede realizarse mediante estudios empíricos que contrastan comportamiento de las técnicas de educción en diferentes contextos.

Este artículo presenta una revisión de literatura sobre los atributos contextuales relevantes para la selección de técnicas de educción. El análisis de esta revisión permitirá en trabajo posterior conocer la coordinación existente entre los expertos que proponen atributos contextuales en base a su experiencia y los experimentadores que realizan estudios para comprobar empíricamente si estos atributos influyen en la efectividad de las técnicas de educción de requisitos software. Los resultados de este estudio pueden contribuir, además, a la planificación de futura investigación empírica en el área de educción de requisitos.

\section{REVISIÓN DE LITERATURA}

La búsqueda de información se basó en una revisión sistemática de artículos de investigación y libros relacionados con las técnicas de educción de requisitos. Para esto, se realizaron búsquedas en tres bases de datos bibliográficas con fecha de inicio ilimitada y fecha final diciembre 2013 (inclusive): Scopus, IEEEXPLORE y ACM DL.

Para la búsqueda en Scopus se utilizó el string: TITLE-ABS-KEY((framework OR comparison OR empirical OR study) AND (requirements OR knowledge) AND (elicitation OR acquisition) AND (techniques OR methods)) AND SUBJAREA (comp OR Undefined).

Para la búsqueda en IEEEXPLORE se utilizó el string de búsqueda: ((framework OR comparison OR empirical OR study) AND (requirements OR knowledge) AND (elicitation OR gathering) AND (techniques OR methods)) AND SUBJECT: Computing \& Processing (Hardware/Software).

Para la búsqueda en ACM DL se utilizó el string de búsqueda: ((Title: framework OR Title: comparison OR Title: empirical OR Title: study) AND (Title: requirements OR Title: knowledge) AND (Title: elicitation OR Title: gathering) AND (Title: techniques OR Title: methods))OR ((Abstract: framework OR Abstract: comparison OR Abstract: empirical OR Abstract: study) AND (Abstract: requirements OR Abstract: knowledge) AND (Abstract: elicitation OR Abstract: gathering) AND (Abstract: techniques OR Abstract: methods)).

Adicionalmente a esta búsqueda, también se realizó una búsqueda no sistemática considerando las referencias bibliográficas de artículos seleccionados, revisiones de libros relacionados con requisitos y búsquedas oportunísticas en Internet. 
La Figura 1 muestra los resultados obtenidos en estas búsquedas. En las búsquedas se consideraron dos tipos de filtros: uno revisando el título y abstract (1F: primer filtro), y otro revisando el artículo completo (2F: segundo filtro).



Figura 1. Metodología de búsqueda.

Para seleccionar los trabajos útiles se tuvieron en cuenta los siguientes aspectos considerados como criterios de filtro:

- Aunque las técnicas de educción de interés son las de SE, es posible considerar estudios sobre otras áreas donde se aplican estas técnicas. Además, las técnicas pueden ser individuales o grupales, es decir, pueden educir información desde uno o varios stakeholders.

- Serán de interés los atributos que tienen relación con el proceso de educción de requisitos. Hay atributos propuestos en la literatura que pueden influir sobre el resto de las actividades de requisitos pero no sobre educción. Por ejemplo, volatilidad de los requisitos y número de requisitos son atributos que pueden ser establecidos una vez que los requisitos son capturados por lo que su influencia es relacionada con las actividades posteriores [12].

- En los estudios empíricos, la efectividad de las técnicas de educción puede ser medida de diferente forma [13]. Esta diversidad de medición de la adecuación de las técnicas puede ser relevante para la agregación de resultados de experimentos pero no es importante para decidir si un atributo influye en la diferenciación de técnicas. Es decir, independientemente de cómo se mida la efectividad de las técnicas en los estudios empíricos, una diferencia de los resultados de aplicar las técnicas bajo las condiciones de un atributo es suficiente para considerarlo como influyente.

- No se consideraron los estudios sobre herramientas de soporte de educción de requisitos debido a que pueden agregar características diferenciadoras importantes a las técnicas evaluadas.

- Los estudios sobre caracterización de las técnicas de educción del tipo [3, 5], en general, no fueron seleccionados ya que se refieren más bien a aspectos intrínsecos, descriptivos o prescriptivos que dependen de la naturaleza de las técnicas, que a aspectos del contexto en que pueden ser aplicadas. Por ejemplo, aspectos descriptivos del tipo "se administran oralmente o escritos", en lugar de aspectos del contexto del tipo "stakeholders está en línea o no". Estos aspectos del contexto (disponibilidad o localización) son los que pueden variar de un caso a otro, incluso entre sesiones de educción. Las características de las técnicas permanecen estables (modalidad o formatos de administración de los cuestionarios) $\mathrm{y}$ aunque son las que sustentan sus bondades y adecuaciones de uso no permiten, por sí solas, diferenciar la efectividad de las técnicas. En otras palabras, se realiza una encapsulación de la técnica como herramienta a utilizar con un éxito relativo dependiendo del contexto en que se pretende utilizar. Por ejemplo, un atributo o característica como habilidad para facilitar la comunicación [14], propiedad de las técnicas, no permite saber en qué condiciones es posible utilizarla. Siempre será necesario establecer las condiciones del entorno para saber si es adecuada o no en ese contexto. Por ejemplo, el atributo del contexto problemas de comunicación de los participantes puede usarse para, en base a él, decidir las técnicas que serán más adecuadas. La habilidad para facilitar la comunicación de una técnica será la que establezca que dicha técnica es propicia para ser aplicada en un contexto donde existen problemas de comunicación.

- No todos los estudios empíricos sobre efectividad sirven para esta investigación. 
En rigor, se centró en aquellos que comparan más de una técnica de educción considerando, además, alguna condición o atributo contextual como variable moderadora o factor. Los resultados de experimentos comparativos permiten saber si hay diferencias de efectividad de las técnicas, detectándose así la influencia del atributo. Un estudio sobre una única técnica no permite aseverar que el atributo o los atributos considerados diferencian la efectividad entre técnicas y por tanto no ayuda en la selección. Por ejemplo, en [15] se estudia el Método Delphi para comparar la información adquirida desde stakeholders individuales y grupales. Aunque los resultados demostraron que es mayor y mejor aquella información obtenida desde grupos es posible que esta efectividad relativa sea similar en todas las técnicas de educción, con lo que no existiría influencia del atributo referido a la cantidad de informantes para diferenciar su adecuación y seleccionar una en lugar de otras. Tampoco es útil un estudio de efectividad de varias técnicas pero sin establecer un factor variable [16-18]. El factor del contexto es precisamente la información relevante para tomar la decisión de qué técnica puede ser más efectiva en un determinado contexto.

- El tipo de diseño de los estudios empíricos (trial, crossover, case study, etc.) no es relevante para esta investigación. El interés se focaliza sobre los resultados y los factores considerados.

- Algunos estudios son catalogados de empíricos o teóricos según el aporte significativo para este estudio. Por ejemplo, en [19] se presenta un estudio empírico sobre una única técnica (event-based elicitation) que, como se explicó arriba no son considerados en este estudio. Sin embargo, además los autores declaran la dependencia de algunas técnicas de factores como el conocimiento del dominio y el momento del proceso. En este caso, este estudio se consideró como teórico ya que su utilidad en esta investigación es de esa naturaleza.

Como se observa en la Figura 1, la revisión de Scopus y la revisión no sistemática fueron las más productivas ya que la gran mayoría de los estudios de interés fueron identificados de esta forma. En Scopus se seleccionaron 23 artículos de un total de 2.962. En búsquedas oportunísticas, libros y otras bibliografías, se encontraron 18 trabajos de interés de un total aproximado de 165 publicaciones. Las otras búsquedas sistemáticas no arrojaron un número importante de nuevas publicaciones a pesar del gran volumen revisado (6 de 420 en IEEEXPLORE y 1 de 257 en ACM DL). Los estudios seleccionados consideran libros, revistas, conferencias, disertaciones, entre otros.

La Tabla 1 muestra estos estudios. Cabe destacar que de los 48 estudios seleccionados, 26 son teóricos y 22 empíricos, y que 19 provienen de la Ingeniería del Conocimiento, 24 de la Ingeniería de Software y 5 de los Sistemas de Información.

\section{ATRIBUTOS PROPUESTOS EN LITERATURA}

Una vez revisadas las 48 publicaciones seleccionadas se identificaron 117 atributos contextuales influyentes en la selección de técnicas de educción. Estos atributos fueron clasificados según el factor contextual al que pertenecen. Se establecieron 5 factores: Eductor (quien realiza la educción), Informante (quien posee la información relevante), Dominio del Problema (área del conocimiento donde se aloja el problema), Dominio de la Solución (disciplina y tecnologías que son necesarias para generar la solución) y Proceso de Educción (actividades y entorno en que se realiza el proceso).

\section{Atributos del Eductor}

De los 118 atributos encontrados, 16 pertenecen a características propias de la persona que realiza la captura de información, denominado normalmente ingeniero de requisitos o analista (14\%). Algunos de estos atributos se repetían o representaban la misma característica por lo que se agruparon bajo un mismo nombre. Los nombres originales se mantuvieron en inglés para mantener total fidelidad. Por ejemplo, los atributos Domain Knowledge y Experience in the Problem Domain and Application Type, de las publicaciones $\mathrm{P} 04$ y P28, respectivamente, por proximidad de definición se reagruparon en el atributo Familiaridad con el Dominio. De esta forma, como muestra la Tabla 2, los 16 atributos quedaron agrupados en 4 atributos genéricos. 
Tabla 1. Publicaciones Seleccionadas.

\begin{tabular}{|c|c|c|c|c|}
\hline Cód. & AÑ̃ & & Origen & ipo \\
\hline P01 & 1988 & Kim and Courtney [20] & Ing. Conoc. & Teórico \\
\hline $\mathrm{P} 02$ & 988 & Grabowski [21] & ng. Conoc. & Empírico \\
\hline P03 & 89 & & toc. & Empírico \\
\hline P04 & 990 & $\mathrm{val}$ and $\mathrm{Be}$ & g. Conoc. & Teórico \\
\hline P05 & 90 & 11 & st. Inform & Empírico \\
\hline P06 & 1990 & Burton y otros & ng. Conoc. & Empírico \\
\hline P07 & 1991 & zlo & g. Softw. & Teórico \\
\hline P08 & 1991 & Ма & ng. Conoc. & Empírico \\
\hline P09 & 1991 & $\mathrm{McCl}$ & ng. Conoc. & Empírico \\
\hline P10 & 1992 & Byrd, C & Ing. Conoc. & Teórico \\
\hline P11 & 1992 & Christel and K. K & Ing. Softw. & Teórico \\
\hline P12 & 992 & & & \\
\hline P13 & 1994 & & ig. Conoc. & \begin{tabular}{|l|} 
Teórico \\
\end{tabular} \\
\hline P14 & 994 & & & En \\
\hline P15 & 1994 & Holsa & ng. Conoc. & Empírico \\
\hline P16 & 1995 & & oc. & Em \\
\hline P17 & 1995 & id $c$ & g. Softw. & Empírico \\
\hline P18 & 1996 & $\mathrm{M}$ & g. $s$ & \\
\hline P19 & 1999 & ody, & g. Conoc. & \\
\hline P20 & 2000 & $\mathrm{~F}$ & g. Conoc. & \\
\hline P21 & 2000 & Moore and $\mathrm{S}$ & $\mathrm{ttw}$. & rico \\
\hline P22 & 2000 & $\mathrm{~s}$ & g. Softw. & Em \\
\hline P23 & 2001 & & $\mathrm{~m}$ & \\
\hline P24 & 2001 & ee an & ist. Inform. & Empírico \\
\hline P25 & 2002 & & g. Softw. & \\
\hline P26 & 2002 & Rosson & g. Softw. & Empírico \\
\hline P27 & 2002 & (me & g. Softw. & Emp \\
\hline P28 & 2003 & Dovic & g. Softw. & Teórico \\
\hline P29 & 2003 & & & \\
\hline P30 & 2003 & & g. Conoc. & Empírico \\
\hline P31 & 2005 & & & \\
\hline P32 & 2005 & & $\mathrm{ftw}$. & ico \\
\hline P33 & 2005 & $20 \mathrm{v}$ & g. Sc & \\
\hline P34 & 2006 & & st. Inform. & Teórico \\
\hline P35 & 2006 & ulin y $\mathrm{c}$ & ng. Conoc. & Teórico \\
\hline P36 & 2006 & 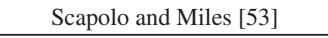 & Sist. Inform & Empírico \\
\hline P37 & 2007 & & g. Softw. & Teórico \\
\hline P38 & 2007 & , & g. Softw. & Teórico \\
\hline P39 & 2008 & Нй 5 & Ing. Conoc. & Teórico \\
\hline $\mathrm{P} 40$ & 2008 & ind Sut & g. Softw. & Teórico \\
\hline P41 & 2008 & lsapple, Raj and Wagner [57] & Ing. Conoc. & Empírico \\
\hline P42 & 2010 & - & Ing. Softw. & Teórico \\
\hline P43 & 2010 & usar y c & Ing. Softw. & Teórico \\
\hline P44 & 2012 & Tiwari, Rathore and Gupta [60] & Ing. Softw. & Teórico \\
\hline P45 & 2012 & Serna [ & g. Softw. & Teórico \\
\hline P46 & 2012 & pata y o & g. Softw. & Empíric \\
\hline P47 & 2012 & & tw. & Empí \\
\hline P48 & 2012 & Rodina, Amjed and Zarinah [64] & Ing. Softw. & Empíri \\
\hline
\end{tabular}

\section{Atributos del Informante}

De los 118 atributos encontrados, 32 pertenecen a características propias de la persona o del grupo de personas que poseen la información relevante para conformar los requisitos, denominados normalmente stakeholders (27\%). Una gran cantidad de atributos
Tabla 2. Atributos del Eductor.

\begin{tabular}{|c|c|c|}
\hline $\begin{array}{l}\text { Atributos } \\
\text { Genéricos }\end{array}$ & Atributos Originales & Pub. \\
\hline \multirow{3}{*}{$\begin{array}{l}\text { Experiencia } \\
\text { Capturando } \\
\text { Información }\end{array}$} & Knowledge Acquisition Experience & P04 \\
\hline & Requirements Elicitation Experience & $\mathrm{P} 26$ \\
\hline & Knowledge Acquisition Experience & $\mathrm{P} 05$ \\
\hline \multirow{3}{*}{$\begin{array}{l}\text { Formación } \\
\text { en Técnicas } \\
\text { de Educción }\end{array}$} & $\begin{array}{l}\text { Technical Knowledge/Exp. with Elicitation } \\
\text { Methods }\end{array}$ & P04 \\
\hline & $\begin{array}{l}\text { Knowledge/Experience with Elicitation } \\
\text { Techniques }\end{array}$ & $\mathrm{P} 28$ \\
\hline & Techniques Training/Knowledge Level & P29 \\
\hline \multirow{6}{*}{$\begin{array}{l}\text { Familiaridad } \\
\text { con el } \\
\text { Dominio }\end{array}$} & Domain Knowledge & P04 \\
\hline & Domain Knowledge & $\mathrm{P} 20$ \\
\hline & Business Knowledge & P34 \\
\hline & $\begin{array}{l}\text { Experience in Problem Domain and } \\
\text { Application Type }\end{array}$ & $\mathrm{P} 28$ \\
\hline & Domain Knowledge & $\mathrm{P} 47$ \\
\hline & Perceived Effects of Domain Knowledge & $\mathrm{P} 47$ \\
\hline \multirow{4}{*}{$\begin{array}{l}\text { Aspectos } \\
\text { Cognitivos }\end{array}$} & Analist Abilities & $\mathrm{P} 29$ \\
\hline & Analyst Ability/Skill & $\mathrm{P} 44$ \\
\hline & Communication/Facilitation Skills & $\mathrm{P} 28$ \\
\hline & Requirements Engineer Type & P31 \\
\hline
\end{tabular}

coincidían en definición o naturaleza por lo que se agruparon bajo un mismo nombre. Por ejemplo, los atributos User Involvement, Stakeholder Involvement, User' Participation Level y Customer Participation, por proximidad de definición se reagruparon en el atributo Participación de Stakeholders. De esta forma, como muestra la Tabla 3, los 32 atributos quedaron agrupados en 6 atributos genéricos.

\section{Atributos del Dominio del Problema}

De los 118 atributos encontrados, 37 pertenecen a características del problema a resolver (31\%). Algunos de estos atributos se repetían entre publicaciones. Por ejemplo, el atributo Knowledge Types es propuesto en las publicaciones $\mathrm{P} 18, \mathrm{P} 23$, P13, P39, P01 y P09. Otros atributos representaban el mismo concepto por lo que se agruparon, también, bajo un mismo nombre. De esta forma, como muestra la Tabla 4, los 37 atributos quedaron agrupados en 6 atributos genéricos.

\section{Atributos del Dominio de la Solución}

De los 118 atributos encontrados, 11 pertenecen a características de la tecnología a desarrollar para dar solución al problema (9\%).

Algunos atributos referenciaban relativamente el mismo concepto por lo que se unieron bajo un mismo nombre de atributo. Por ejemplo, el atributo Representation of Knowledge y Methods of Resolution se reunieron en el atributo Métodos de Resolución. 
Tabla 3. Atributos del Informante.

\begin{tabular}{|c|c|c|}
\hline $\begin{array}{l}\text { Atributos } \\
\text { Genéricos }\end{array}$ & Atributos Originales & Publ. \\
\hline \multirow{4}{*}{$\begin{array}{l}\text { Número de } \\
\text { Informantes }\end{array}$} & Number of Stakeholders & P18 \\
\hline & Number of Stakeholders & $\mathrm{P} 43$ \\
\hline & Stakeholder Count & $\mathrm{P} 28$ \\
\hline & Experts Number & $\mathrm{P} 08$ \\
\hline \multirow{4}{*}{$\begin{array}{l}\text { Participación de } \\
\text { Stakeholders }\end{array}$} & User Involvement & $\mathrm{P} 31$ \\
\hline & Stakeholder Involvement & $\mathrm{P} 43$ \\
\hline & User' Participation Level & P29 \\
\hline & Customer Participation & $\mathrm{P} 26$ \\
\hline \multirow{6}{*}{$\begin{array}{l}\text { Aspectos } \\
\text { Geográficos }\end{array}$} & Temporal Co-Location & $\mathrm{P} 28$ \\
\hline & Physical Co-Location & $\mathrm{P} 28$ \\
\hline & Culture Diversity & P37 \\
\hline & Cultural Diversity & $\mathrm{P} 27$ \\
\hline & Time Difference & $\mathrm{P} 27$ \\
\hline & Synchronization & $\mathrm{P} 26$ \\
\hline \multirow{5}{*}{$\begin{array}{l}\text { Fuente de } \\
\text { Información }\end{array}$} & Stage of Expertise & $\mathrm{P} 04$ \\
\hline & Type of End Users & $\mathrm{P} 44$ \\
\hline & Source of Requirements & $\mathrm{P} 45$ \\
\hline & Type of Stakeholders & $\mathrm{P} 44$ \\
\hline & Level of Expertise & P06 \\
\hline \multirow{2}{*}{$\begin{array}{l}\text { Capacidad de } \\
\text { Articulación }\end{array}$} & Articulability Difficulties & $\mathrm{P} 34$ \\
\hline & Verbalizations of Knowledge & $\mathrm{P} 03$ \\
\hline \multirow{11}{*}{$\begin{array}{l}\text { Aspectos } \\
\text { Personales }\end{array}$} & Cognitive Styles & $\mathrm{P} 04$ \\
\hline & Personality Variables & $\mathrm{P} 04$ \\
\hline & Motivations & $\mathrm{P} 40$ \\
\hline & Values & $\mathrm{P} 40$ \\
\hline & Emotions & $\mathrm{P} 40$ \\
\hline & Personal Values & $\mathrm{P} 42$ \\
\hline & Cognitive Limitations & $\mathrm{P} 37$ \\
\hline & Communication Obstacles & $\mathrm{P} 10$ \\
\hline & Stakeholders Categories & $\mathrm{P} 32$ \\
\hline & Personality Characteristics & $\mathrm{P} 14$ \\
\hline & Cognitive Abilities & P16 \\
\hline
\end{tabular}

De esta forma, como muestra la Tabla 5, los 11 atributos quedaron agrupados en 3 atributos genéricos.

\section{Atributos del Proceso}

Finalmente, de los 118 atributos encontrados, 22 pertenecen a características del proceso de captura de información para especificar requisitos (19\%).

Algunos de estos atributos se agruparon en una categoría superior de abstracción. Por ejemplo, los atributos Time/Cost Constraints, Budget Constraints, Schedule Constraints, Resource Constraints, Time Constraints, Barriers in Communication y Cost Constraints, se agruparon bajo el atributo genérico Restricciones del Proceso. Otros atributos representaban el mismo concepto, como: Versions,
Tabla 4. Atributos del Dominio del Problema.

\begin{tabular}{|c|c|c|}
\hline $\begin{array}{l}\text { Atributos } \\
\text { Genéricos }\end{array}$ & Atributos Originales & Publ. \\
\hline \multirow{16}{*}{$\begin{array}{l}\text { Tipos de } \\
\text { Información }\end{array}$} & Observable Phenomena & P18 \\
\hline & Knowledge Types & P18 \\
\hline & Requirements Abstraction Level & P37 \\
\hline & Information Types & $\mathrm{P} 25$ \\
\hline & Knowledge Types & $\mathrm{P} 23$ \\
\hline & Problem Domain Categories & $\mathrm{P} 10$ \\
\hline & Knowledge Types & $\mathrm{P} 13$ \\
\hline & Types of Knowledge & P39 \\
\hline & Human Knowledge Categories & P19 \\
\hline & Internal Filtering of Knowledge & P18 \\
\hline & Types of Knowledge & $\mathrm{P} 01$ \\
\hline & Generic Requirements Categories & $\mathrm{P} 24$ \\
\hline & Types of Knowledge and Information & $\mathrm{P} 36$ \\
\hline & Heuristics Types & $\mathrm{P} 02$ \\
\hline & Knowledge Types & P09 \\
\hline & Types of Requirements & $\mathrm{P} 21$ \\
\hline \multirow{3}{*}{$\begin{array}{l}\text { Tipos de } \\
\text { Dominios }\end{array}$} & Domains Types & P06 \\
\hline & Type of Application Domain & $\mathrm{P} 35$ \\
\hline & Domain Stability & P31 \\
\hline \multirow{7}{*}{$\begin{array}{l}\text { Grado de } \\
\text { Definición }\end{array}$} & Uncertainty Degree & P04 \\
\hline & Fuzziness of Definition & $\mathrm{P} 28$ \\
\hline & Unknown Domain Degree & P34 \\
\hline & Certainty Level & $\mathrm{P} 45$ \\
\hline & Level of Certainty & P37 \\
\hline & Structuredness & $\mathrm{P} 01$ \\
\hline & Uncertainty/Equivocality Degree & P07 \\
\hline \multirow{3}{*}{$\begin{array}{l}\text { Tipos de } \\
\text { Tareas }\end{array}$} & Tasks Types & $\mathrm{P} 04$ \\
\hline & Problem Types & $\mathrm{P} 30$ \\
\hline & Scenarios & P08 \\
\hline \multirow{3}{*}{$\begin{array}{l}\text { Tamaño del } \\
\text { Problema }\end{array}$} & Number of Elements & $\mathrm{P} 01$ \\
\hline & Project Size & P38 \\
\hline & Information Resource Amount & $\mathrm{P} 31$ \\
\hline \multirow{5}{*}{ Complejidad } & Number of Interrelationships & $\mathrm{P} 01$ \\
\hline & Project Complexity & P38 \\
\hline & Complexity & $\mathrm{P} 28$ \\
\hline & Domain Complexity & P41 \\
\hline & Domain Complexity & $\mathrm{P} 15$ \\
\hline
\end{tabular}

Tabla 5. Atributos del Dominio de la Solución.

\begin{tabular}{|l|l|c|}
\hline $\begin{array}{l}\text { Atributos } \\
\text { Genéricos }\end{array}$ & \multicolumn{1}{|c|}{ Atributos Originales } & Publ. \\
\hline \multirow{4}{*}{$\begin{array}{l}\text { Tipos de } \\
\text { Productos }\end{array}$} & Project Category & P38 \\
\cline { 2 - 3 } & Type of Solution & P04 \\
\cline { 2 - 3 } & Target Stakeholder & P43 \\
\cline { 2 - 3 } & Project Status & P43 \\
\cline { 2 - 3 } & Domain of System being Developed & P44 \\
\cline { 2 - 3 } & Scope of System & P44 \\
\cline { 2 - 3 } & Type of Project & P43 \\
\cline { 2 - 3 } & Development Environments & P17 \\
\hline \multirow{3}{*}{$\begin{array}{l}\text { Grado de } \\
\text { Criticidad }\end{array}$} & Degree of Safety Criticality & P38 \\
\hline $\begin{array}{l}\text { Métodos de } \\
\text { Resolución }\end{array}$ & Representation of Knowledge & P19 \\
\cline { 2 - 3 } & Methods of Resolution \\
\hline
\end{tabular}


Communication Mode y Conference Types por lo que se agruparon, también, bajo Tipos de Conferencias.

De esta forma, como muestra la Tabla 6, los 22 atributos quedaron agrupados en 8 atributos genéricos.

\section{ATRIBUTOS GENÉRICOS DE INFLUENCIA}

En resumen, los 118 atributos encontrados en literatura dieron lugar a 27 atributos genéricos que influyen en la selección de la técnica de educción más adecuada a un contexto de proyecto software.

Estos atributos agrupados por factor se muestran en la Tabla 7. El factor que centró más atención fue el Dominio del Problema, con 37 atributos, seguido del Informante con 32. El Dominio de la Solución fue el menos tratado con solo 11 atributos propuestos.

Tabla 6. Atributos del Proceso.

\begin{tabular}{|c|c|c|}
\hline $\begin{array}{l}\text { Atributos } \\
\text { Genéricos }\end{array}$ & Atributos Originales & Pub. \\
\hline \multirow{2}{*}{$\begin{array}{l}\text { Propósito de los } \\
\text { Requerimientos }\end{array}$} & Purpose of Requirements & P18 \\
\hline & Level of Abstraction & $\mathrm{P} 45$ \\
\hline Entregable & Deliverable Type & P35 \\
\hline $\begin{array}{l}\text { Entorno del } \\
\text { Proyecto }\end{array}$ & Social Environment & $\mathrm{P} 44$ \\
\hline \multirow{2}{*}{$\begin{array}{l}\text { Tipo Proyecto } \\
\text { de Educción }\end{array}$} & Type of Elicitation Project & P35 \\
\hline & Software Development Scenarios & $\mathrm{P} 46$ \\
\hline \multirow{4}{*}{$\begin{array}{l}\text { Tipos de } \\
\text { Conferencias }\end{array}$} & Versions & P14 \\
\hline & Versions & P12 \\
\hline & Communication Mode & $\mathrm{P} 48$ \\
\hline & \begin{tabular}{|l|} 
Conference Types \\
\end{tabular} & $\mathrm{P} 22$ \\
\hline \multirow{7}{*}{$\begin{array}{l}\text { Restricciones } \\
\text { del Proceso }\end{array}$} & Time/Cost Constraints & P18 \\
\hline & Budget Constraints & $\mathrm{P} 43$ \\
\hline & Schedule Constraints & $\mathrm{P} 43$ \\
\hline & Resource Constraints & $\mathrm{P} 43$ \\
\hline & Time Constraints & P38 \\
\hline & Barriers in Communication & $\mathrm{P} 45$ \\
\hline & Cost Constraints & P38 \\
\hline \multirow{3}{*}{$\begin{array}{l}\text { Momento del } \\
\text { Proceso }\end{array}$} & Activities & P33 \\
\hline & \begin{tabular}{|l|} 
Job Analysis \\
\end{tabular} & P20 \\
\hline & Early Tasks & P11 \\
\hline \multirow{2}{*}{ Metodologías } & Approach to be Followed out & P44 \\
\hline & System Development Methodology & P04 \\
\hline
\end{tabular}

Esta reducción significativa demuestra que hay poca uniformidad de lenguaje entre los investigadores de las técnicas de educción de requisitos y que una ontología unificada sobre estos atributos es totalmente necesaria.

\section{CONCLUSIONES Y TRABAJO FUTURO}

En este artículo se presentó una revisión sistemática de literatura sobre los atributos contextuales que influyen en la efectividad de las técnicas de educción de requisitos. Esta revisión fue complementada con una revisión no sistemática. Estas revisiones tenían por objetivo seleccionar los estudios que proponían atributos contextuales y aquellos que los utilizaban como factores en sus experimentos.

Tabla 7. Atributos Genéricos por Factor.

\begin{tabular}{|c|c|}
\hline Factor & Atributos Genéricos \\
\hline \multirow[t]{4}{*}{ Eductor } & Experiencia en Captura de Información \\
\hline & Formación en Técnicas de Educción \\
\hline & Familiaridad con el Dominio \\
\hline & Aspectos Cognitivos \\
\hline \multirow{6}{*}{ Informante } & Número de Informantes \\
\hline & Participación de Stakeholders \\
\hline & Aspectos Geográficos \\
\hline & Fuente de Información \\
\hline & Capacidad de Articulación \\
\hline & Aspectos Personales \\
\hline \multirow{6}{*}{$\begin{array}{l}\text { Dominio } \\
\text { del } \\
\text { Problema }\end{array}$} & Tipos de Información \\
\hline & Tipos de Dominios \\
\hline & Grado de Definición \\
\hline & Tipos de Tareas \\
\hline & Tamaño del Problema \\
\hline & Complejidad \\
\hline \multirow{3}{*}{$\begin{array}{l}\text { Dominio } \\
\text { de la } \\
\text { Solución }\end{array}$} & Tipos de Productos \\
\hline & Grado de Criticidad \\
\hline & Métodos de Resolución \\
\hline \multirow{8}{*}{$\begin{array}{l}\text { Proceso de } \\
\text { Educción }\end{array}$} & Propósito de los Requerimientos \\
\hline & Entregable \\
\hline & Entorno del Proyecto \\
\hline & Tipo de Proyecto de Educción \\
\hline & Tipos de Conferencias \\
\hline & Restricciones del Proceso \\
\hline & Momento del Proceso \\
\hline & Metodologías \\
\hline
\end{tabular}

El estudio arrojó sobre un centenar de atributos distribuidos sobre cinco factores contextuales relevantes en el proceso de captura de información relevante para conformar los requisitos software. Estos atributos se consolidan, no obstante, en cerca de una treintena de atributos genéricos que representan aspectos contextuales influyentes sobre la efectividad de la educción de requisitos. Esta diversidad pone de manifiesto la gran sensibilidad 
que puede tener el comportamiento de las técnicas de educción en un caso particular.

El trabajo futuro a desarrollar contempla en examinar la coordinación entre lo que creen los investigadores y lo que realmente estudian empíricamente. Esto, con el fin de contribuir con una orientación sobre qué factores considerar en experimentación en requisitos.

\section{REFERENCIAS}

[1] A. Abran and J.W. Moore. "SWEBOK: Guide to the Software Engineering Body of Knowledge (2004 edition)". 2004. Date of visit: May 30, 2005. URL: http://www. computer.org/portal/web/swebok

[2] H. Beyer and K. Holtzblatt. "Apprenticing with the Customer". Communications of the ACM. Vol. 38, Issue 5, pp. 45-52. May, 1995.

[3] J. Goguen and C. Linde. "Techniques for requirements elicitation". International Symposium on Requirements Engineering. Los Alamitos, California: IEEE Computer Society Press, pp. 152-164. January, 1993.

[4] P.B. Nuseibeh and S.M. Easterbrook. "Requirements engineering: A roadmap". The Future of Software Engineering. A. C.W. Finkelstein, Ed. (Companion volume to the proceedings of the 22nd International Conference on Software Engineering, ICSE'00). IEEE Computer Society Press, 2000.

[5] N. Cooke. "Varieties of knowledge elicitation techniques". International Journal of Human Computer Studies. Vol. 41, Issue 6, pp. 801849. December, 1994.

[6] R. Hoffman, N. Shadbolt, A.M. Burton and G.A. Klein. "Eliciting knowledge from experts: A methodological analysis". Organizational Behavior and Human Decision Processes. Vol. 62, pp. 129-158. 1995.

[7] H. Saiedian and R. Dale. "Requirements engineering: making the connection between the software developer and customer". Information and Software Technology. Vol. 42, Issue 6, pp. 419-28. April, 2000.

[8] L. Macaulay. "Requirements for requirements engineering techniques". Proceedings of the Second International Conference on Requirements Engineering (Cat. $\mathrm{N}^{\circ}$ 96TB100037). IEEE Comput. Soc. Press, pp. 157-64. Los Alamitos, CA, USA. 1996.

[9] S. Robertson and J. Robertson. "Mastering the requirements process". Addison-Wesley. 1999.

[10] A. Davis and A. Hickey. "Requirements Researchers: Do We Practice What We Preach". Requirements Engineering Journal. Vol. 7, Issue 2, pp. 107-111. 2002.

[11] D. Zowghi and C. Coulin. "Requirements Elicitation: A Survey of Techniques, Approaches, and Tools”. In: Book chapter in A. Aurum, and C. Wohlin (Eds.), Engineering and Managing software requirements. Springer-Verlag, pp. 19-46. New York, USA. 2005.

[12] Li Jiang, A. Eberlein and B.H. Far. "A case study validation of a knowledge-based approach for the selection of requirements engineering techniques". Requirements Engineering Journal. Vol. 13, Issue 2, pp. 117-146. 2008.

[13] O. Dieste and N. Juristo. "Systematic Review and Aggregation of Empirical Studies on Elicitation Techniques". IEEE Transactions on Software Engineering. DOI: 10.1109/ TSE.2010.33. 2010.

[14] E. Kheirkhah and A. Deraman. "Important factors in selecting Requirements Engineering Techniques". International Symposium on Information Technology. 2008.

[15] M. Roth and C. Wood. "Knowledge acquisition from single versus multiple experts: a field study comparison using the Delphi technique". The journal of Knowledge Engineering. Vol. 6, Issue 3. Fall 1993.

[16] S. Jones, J. Miles and M. Read. "A comparison of knowledge elicitation methods". Expert Systems. Vol. 13, Issue 4, pp. 277-95. Nov. 1996.

[17] E. Hudlicka. "Requirements Elicitation with Indirect Knowledge Elicitation Techniques: Comparison of Three Methods". Second IEEE International Conference on Requirements Engineering, Los Alamitos, California: IEEE Computer Society Press. April, 1996.

[18] L. Adelman. "Measurement issues in knowledge engineering". IEEE Transactions on Systems, Man and Cybernetics. Vol. 19, pp. 483-488. 1989. 
[19] J.E. Fowlkes, E. Salas and D.P. Baker. "The utility of event-based knowledge elicitation". Human Factors. Vol. 42, pp. 24-35. 2000.

[20] J. Kim and J. Courtney. "A survey of knowledge acquisition techniques and their relevance to managerial problem domains". Decision Support Systems. Vol. 4, pp. 269284. 1988.

[21] M. Grabowski. "Knowledge acquisition methodologies: survey and empirical assessment". Proceedings of the Ninth International Conference on Information Systems, pp. 47-54. 1988.

[22] B. Crandall. "A comparative study of think aloud and critical decision knowledge elicitation methods". SIGART Newsletter. Vol. 108, pp. 144-146. 1989.

[23] J.S. Dhaliwal and I. Benbazat. "A framework for the comparative evaluation of knowledge acquisition tools and techniques". Knowledge Acquisition. Vol. 2, Issue 2, pp. 145-166. June, 1990.

[24] R. Agarwal and M. Tanniru. "Knowledge acquisition using structured interviewing: an empirical investigation". Journal of Management Information Systems. Vol. 7, Issue 1, pp. 123-140. Summer 1990.

[25] A. Burton, N. Shadbolt, G. Rugg and A. Hedgecock. "The efficacy of knowledge elicitation techniques: a comparison across domains and levels of expertise". Knowledge Acquisition. Vol. 2, Issue 2, pp. 167-178. June, 1990.

[26] B. Fazlollahi and M. Tanniru. "Selecting a requirements determination methodologycontingency approach revisited". Information and Management. Vol. 21, Issue 5, pp. 291303. 1991.

[27] A.P. Massey and W.A. Wallace. "Focus groups as a knowledge elicitation technique: an exploratory study". IEEE Transactions on Knowledge and Data Engineering. Vol. 3 Issue 2, pp. 193-200. 1991.

[28] B.P. McCloskey, J. Geiwitz and J. Kornell. "Empirical comparisons of knowledge acquisition techniques". Proc. of the Human Factors Society 35 th Annual Meeting Human Factors Soc. Vol. 1, pp. 268-272. Santa Monica, CA, USA. 1991.

[29] T.A. Byrd, K.L. Cossick and R.W. Zmud. "A synthesis of research on requirements analysis and knowledge acquisition techniques". MIS Quarterly. Vol. 16, pp. 117-138. 1992.

[30] M. Christel and K. Kang. "Issues in Requirements Elicitation". SEI Technical Report N ${ }^{\circ}$ SEI-92-TR-012, Carnegie Mellon Software Engineering Institute. September, 1992.

[31] G. Rugg, C. Corbridge, N. Major, A. Burton and N. Shadbolt. "A comparison of sorting techniques in knowledge acquisition". Knowledge Acquisition. Vol. 4, Issue 3, pp. 279-291. September, 1992.

[32] S. Skidmore. "Introducing Systems Analysis". NCC/Blackwell, Manchester. pp. 74-85. 1994.

[33] B. Corbridge, G. Rugg, N.P. Major, N.R. Shadbolt and A.M. Burton. "Laddering - technique and tool use in knowledge acquisition". Knowledge Acquisition. Vol. 6, pp. 315-341. 1994.

[34] C. Holsapple and V. Raj. "Exploratory study of two KA methods". Expert Systems. Vol. 11, Issue 2, pp. 77-87. 1994.

[35] C.J. Chao and G. Salvendy. "Impact of cognitive abilities of experts on the effectiveness of elicited knowledge". Behaviour and Information Technology. Vol. 14, Issue 3, pp. 174-182. May-June, 1995.

[36] M. Keil and E. Carmel. "Customer-developer links". Communications of the ACM. Vol. 38, Issue 5, pp. 33-44. May, 1995.

[37] N. Maiden and G. Rugg. "ACRE: selecting methods for requirements acquisition". Software Engineering Journal. Vol. 11, Issue 3, pp. 183-192. May, 1996.

[38] J.W. Moody, J.E. Blanton and P.H. Cheney. "A theoretically grounded approach to assist memory recall during information requirements determination". Journal of Management Information Systems. Vol. 15, Issue 1, pp. 79-98. Summer 1998.

[39] J.M. Moore and F.M.I Shipman. "A comparison of questionnaire based and GUI based requirements gathering". Proceedings of the Fifteenth IEEE International Conference on Automated Software Engineering, pp. 35-43. 2000.

[40] J. Sauer, S. Schramme and B. Ruttinger. "Knowledge acquisition in ecological product design: the effects of computer-mediated 
communication and elicitation method". Behaviour and Information Technology. Vol. 19, Issue 5, pp. 315-327. October, 2000.

[41] M. Eva. "Requirements acquisition for rapid applications development". Information \& Management. Vol. 39, pp. 101-107. December, 2001.

[42] G.J. Browne and M.B. Rogich. "An empirical investigation of user requirements elicitation: comparing the effectiveness of prompting techniques". Journal of Management Information Systems. Vol. 17, Issue 4, pp. 223-249. Spring 2001.

[43] S. Lauesen. "Software requirements: Styles and techniques". Addison-Wesley. 2002.

[44] W.J. Lloyd, M.B. Rosson and J.D. Arthur. "Effectiveness of elicitation techniques in distributed requirements engineering". Proceedings IEEE Joint International Conference on Requirements Engineering. 2002.

[45] D. Damian and D. Zowghi. "The impact of stakeholders geographical distribution on managing requirements in a multi-site organization". IEEE Joint International Conference on Requirements Engineering, RE'02. Essen, Germany, pp. 319-328. 2002.

[46] A. Davis and A. Hickey. "A tale of two ontologies: The basis for systems analysis technique selection". Proceedings 9th Annual American Conference on Information System. 2003.

[47] E. Batista and A. Carvalho. "Uma Taxonomia Facetada para Técnicas de Elicitação de Requisitos". Anais do WER03 - Workshop em Engenharia de Requisitos, pp. 48-62. Piracicaba-SP, Brasil. Novembro 27-28, 2003.

[48] W. Wagner, Q. Chung and M. Najdawi. "The impact of problem domains and knowledge acquisitions techniques: a content analysis of P/OM expert system case studies". Expert Systems with Applications. Vol. 24, pp. 79-86. 2003.

[49] T. Tsumaki and T. Tamai. "Framework for matching requirements elicitation techniques to project characteristics". Software Process Improvement and Practice. Vol. 11, Issue 5, pp. 505-519. 2006.

[50] G.N. Aranda, A. Vizcaino, A. Cechich and M. Piattini. "Choosing groupware tools and elicitation techniques according to stakeholders' features". ICEIS 2005 International Conference on Enterprise Information Systems. Miami. Proceedings of the Seventh International Conference on Enterprise Information Systems. Vol. 3, pp. 68-75. 2005.

[51] C.J. Davis, R.M. Fuller, M.C. Tremblay and D.J. Berndt. "Communication challenges in requirements elicitation and the use of the repertory grid technique". Journal Comp. Information System. Vol. 46, Issue 5, pp. 78-86. 2006.

[52] C. Coulin, D. Zowghi and A. Sahraoui. "A situational method engineering approach to requirements elicitation workshops in the software development process". Software Process Improvement and Practice. Vol. 11, Issue 5, pp. 451-464. 2006.

[53] F. Scapolo and I. Miles. "Eliciting experts' knowledge: A comparison of two methods". Original Research Article Technological Forecasting and Social Change. Vol. 73, Issue 6, pp. 679-704. July, 2006.

[54] Z. Zhang. "Effective Requirements Development - A Comparison of Requirements Elicitation Techniques". Software Quality Management XV: Software Quality in the Knowledge Society, E. Berki, J. Nummenmaa, I. Sunley, M. Ross and G. Staples (Ed.) British Computer Society, pp. 225-240. 2007.

[55] J. Hua. "Study on knowledge acquisition techniques". Proceedings 2nd International Symposium on Intelligent Information Technology Application. IITA 2008. Vol. 1, Art. $N^{\circ}$ 4739560, pp. 181-185. 2008.

[56] S. Thew and A. Sutcliffe. "Investigating the Role of 'Soft Issues' in the RE Process". Proceedings of 16th IEEE International Requirements Engineering Conference. IEEE Computer Society. Los Alamitos, CA, USA. 2008.

[57] C.W. Holsapple, W. Clyde, V. Raj and S. Victor. "Exploratory study of two KA methods". Expert Systems. Vol. 11, Issue 2, pp. 77-87. 1994.

[58] R. Proynova, B. Paech, A. Wicht and T. Wetter. "Use of personal values in requirements engineering - A research preview". Lecture Notes in Computer Science 6182 LNCS, pp. 17-22. 2010. 
[59] S. Kausar, S. Tariq, S. Riaz and A. Khanum. "Guidelines for the selection of elicitation techniques 6th International Conference on Emerging Technologies (ICET), pp. 265-269. 2010.

[60] S. Tiwari, S. Rathore and A. Gupta. "Selecting requirement elicitation techniques for software projects". Sixth International Conference on Software Engineering (CONSEG), pp. 1-10. 2012. DOI: 10.1109/ CONSEG.2012.6349486.

[61] M.E. Serna. "Analysis and selection to requirements elicitation techniques". 7th Colombian Computing Congress (CCC), pp. 1-7. 2012. DOI: 10.1109/ ColombianCC.2012.6398 001.

[62] S.Zapata, E. Torres, G. Sevilla, L. Aballay and M. Reus. "Effectiveness of traditional software requirement elicitation techniques applied in distributed software development scenarios". XXXVIII Conferencia Latinoamericana Informática (CLEI), pp. 1-7. 2012. DOI: 10.1109/CLEI.2012.6427200.

[63] I. Hadar, P. Soffer and K. Kenzi. "The role of domain knowledge in requirements elicitation via interviews: an exploratory study". Requirements Engineering Journal. Vol. 19, Issue 2, pp. 143-159. 2014. ISSN: 0947-3602.

[64] A. Rodina, T. Amjed and M.K. Zarinah. "An empirical assessment of the use of different communication modes for requirement elicitation and negotiation using students as a subject". IEEE Symposium on Computers and Informatics, ISCI. Art. $\mathrm{N}^{\circ} 6222669$, pp. 70-74. 2012. 